\title{
Allelic imbalance of multiple sclerosis susceptibility genes IKZF3 and IQGAP1 in human peripheral blood
}

\author{
Pankaj K. Keshari ${ }^{1,2^{*}}$, Hanne F. Harbo ${ }^{1,2}$, Kjell-Morten Myhr ${ }^{3,4}$, Jan H. Aarseth ${ }^{3,4}$, Steffan D. Bos ${ }^{1,2 \dagger}$ and Tone Berge ${ }^{1 \dagger}$
}

\begin{abstract}
Background: Multiple sclerosis is a chronic inflammatory, demyelinating disease of the central nervous system. Recent genome-wide studies have revealed more than 110 single nucleotide polymorphisms as associated with susceptibility to multiple sclerosis, but their functional contribution to disease development is mostly unknown.

Results: Consistent allelic imbalance was observed for rs907091 in IKZF3 and rs11609 in IQGAP1, which are in strong linkage disequilibrium with the multiple sclerosis associated single nucleotide polymorphisms rs12946510 and rs8042861, respectively. Using multiple sclerosis patients and healthy controls heterozygous for rs907091 and rs11609, we showed that the multiple sclerosis risk alleles at IKZF3 and IQGAP1 are expressed at higher levels as compared to the protective allele. Furthermore, individuals homozygous for the multiple sclerosis risk allele at IQGAP1 had a significantly higher total expression of IQGAP1 compared to individuals homozygous for the protective allele.

Conclusions: Our data indicate a possible regulatory role for the multiple sclerosis-associated IKZF3 and IQGAPI variants. We suggest that such cis-acting mechanisms may contribute to the multiple sclerosis association of single nucleotide polymorphisms at IKZF3 and IQGAPI.
\end{abstract}

Keywords: Allele specific expression, eQTL, Multiple sclerosis, CD69, IKZF3, IQGAP1

\section{Background}

Multiple sclerosis (MS) is a chronic inflammatory disorder of the central nervous system (CNS). Hallmarks of the disease are inflammatory demyelination and axonal loss in the CNS, resulting in neurological dysfunction, typically starting in early adulthood [1]. The cause of the disease is largely unknown, but genetic and environmental factors, as well as interaction of these contribute to disease development [2]. The strongest genetic factor in MS, the Human Leukocyte Antigen ( $H L A)$ gene region, was identified already in 1972 [3], and HLA-DRB1*15:01 displays the strongest genetic signal [4]. Through recent large genome - wide association studies (GWASs) followed up by genome-wide analyses of immune-related loci, more than 110 non-HLA genetic variants were

\footnotetext{
* Correspondence: keshari.pankaj@gmail.com

${ }^{\dagger}$ Equal contributors

'Department of Neurology, Oslo University Hospital, Oslo, Norway

${ }^{2}$ Institute of Clinical Medicine, University of Oslo, Oslo, Norway

Full list of author information is available at the end of the article
}

shown to be associated with MS susceptibility $[4,5]$. The majority of the MS-associated single nucleotide polymorphisms (SNPs) identified through GWAS are located in or near genes having a substantial role in the immune system $[4,6]$.

An important aim of human genetic research is identification of heritable variation in cis-regulatory elements for gene expression that might influence disease risk [7]. The majority (93\%) of the disease susceptibility variants identified through large-scale genetic screens such as GWAS and the immunochip project is located within non-coding regions of the genome [8]. For MS, an enrichment of disease-associated variants is observed in DNase hypersensitive sites in immune cells, especially for $\mathrm{T}$ and $\mathrm{B}$ cells $[8,9]$. Genetic variants may therefore have an influence on the expression of target genes, and the MS-association of the polymorphisms identified through the genetic screens is likely to be the result of such regulatory properties. However, the functional implications of the genetic variants associated with MS are 
largely unknown. To address this, follow-up studies of these MS associated loci are needed to gain insights in the underlying molecular mechanisms. Analysis to unravel whether MS-associated SNPs exert cis-acting regulation is now an important next step in MS research. Such insights will contribute to improved understanding of disease aetiology and may allow development of novel biomarkers and therapies for MS.

The Genotype-Tissue Expression database (GTEx) is providing a valuable tool to investigate genotypedependent gene regulation. The use of a great variety of tissues from human post-mortem donors allows comparing putative genotype-dependent gene regulation between at least 44 tissues in the publicly available pilot data [10]. Data from expression quantitative trait locus (eQTL) databases such as the GTEx is generally based on analyses in heterogeneous groups of individuals, however in specific diseases, gene regulation may be altered due to a disturbed homeostasis. For MS, it is proposed that whole blood composition and immune cell activity is different from healthy controls $[11,12]$. In the current study, we have investigated the allele - specific expression (ASE) of MS-associated SNPs or proxies thereof in whole blood from a cohort of Norwegian MS patients. The unequal output of gene transcript, allelic imbalance (AI), has been established as a ubiquitous phenomenon that may underlie disease risk exerted by disease-associated SNPs [13]. AI may occur when the SNP-containing sequence is within a cis-regulatory region of a gene. A prerequisite to study putative effects on allelic output for disease associated SNPs is that the SNP itself, or a proxy SNP on the same haplotype as the susceptibility SNP, is transcribed. The per-allele expression levels for a transcribed SNP can then be determined in individuals heterozygous for the studied SNP.

Here, we investigate three SNPs in MS associated regions for the presence of AI; (i) the MS-associated SNP rs11052877, which lies in the coding region of $C D 69$, (ii) the rs907091 SNP in the coding region of IKZF3 and in strong linkage disequilibrium (LD) with the MSassociated rs12946510 and (iii) the rs11609 SNP located in the coding region of IQGAPI and in strong LD with the MS-associated rs8042861. Although the data from the GTEx database is extensive, there are examples of genotype-expression correlations not detected. Interestingly, GTEx reports no effect of rs907091 genotype on IKZF3 expression, whereas Fahr and colleagues [14] refer to rs907091 as an eQTL for IKZF3 in lymphoblastoid cell lines [15]. Furthermore, rs11609 in IQGAP1 is reported as an eQTL for IQGAP1 in a wide variety of tissues, but not in whole blood, whereas no SNPs are reported as eQTLs for CD69 in any of the tissues (GTEx, accessed March 2016). In the current analyses, ASE measurements for three SNPs in these three genes (CD69, IKZF3 and IQGAP1) were performed in whole blood samples from Norwegian MS patients and healthy controls.

\section{Methods \\ Subjects, sample collection and reverse transcription of RNA}

Blood samples were collected for DNA (EDTA tubes, Greiner Bio-One, Frickenhausen, Germany) and RNA isolation (Tempus tubes, Applied Biosystems, Foster City, CA, USA) from 140 MS patients collected in the Norwegian multiple sclerosis registry and biobank [16] and 46 healthy controls (14 for RNA extraction) recruited among hospital employees. The MS patients are representative of the Norwegian MS patient population [17]. DNA isolations were performed using DNA isolation columns, and RNA was extracted from Tempus tubes with the Tempus Spin RNA Isolation Kit protocol (Applied Biosystems), including DNase treatment during extraction. The concentration of all DNA and RNA samples was assessed by a nanodrop 2000c spectrophotometer (Thermo Fisher Scientific Inc., Madison, WI, USA). An RNA integrity number above 7.0 of a random set of these RNA samples was verified using the Agilent 2100 Bioanalyzer (Agilent Technologies, Santa Clara, CA, USA). For DNA samples that were used for sensitivity analyses of assays for ASE analyses (see below), the concentration was measured by Qubit Broad Range DNA spectroscopy (Qubit, Life Technologies, Carlsbad, CA, USA). According to manufacturers' protocol, $200 \mathrm{ng}$ RNA was reverse transcribed (RT) with Maxima First Strand complementary DNA (cDNA) synthesis Kit for quantitative polymerase chain reaction (qPCR) (Thermo Scientific, Pittsburgh PA, USA) in a $20 \mu$ reaction.

\section{Single nucleotide polymorphisms included for allele- specific expression analysis}

We used the online tools SNAP for identifying proxy SNPs for the MS associated SNPs [18] and SNPper [19] for determining which of these proxies were transcribed for all 110 non-HLA MS-associated SNPs recently reported $[4,5]$. Three genes, which had a transcribed MSassociated SNP or transcribed proxy for an MSassociated SNP were selected for ASE analyses; CD69, with the MS-associated SNP rs11052877 in its coding region, and IKZF3 and IQGAP1, where rs907091 and rs11609, respectively, are in strong $L D\left(r^{2} \geq 0.8\right)$ with MS-associated SNPs. To verify that the SNP-containing regions were expressed in whole blood, PCRs followed by agarose gel electrophoresis were done on cDNA generated from whole blood. Primer sequences are provided in Additional file 1: Table S1. 


\section{Genotyping}

Genotyping of rs11052877, rs907091, rs11609, rs12946510 and rs8042861 in DNA from the MS patients and healthy controls included those for the Western blot analyses (rs11609 only) was done with TaqMan genotyping assays (C_32169538_10, C_7452266_20, C_26774507_10, C_31651862_10 and C_12091833_10 respectively (Applied Biosystems)). The genotyping reactions were carried out in $5 \mu \mathrm{l}$ using $12 \mathrm{ng}$ of genomic DNA (gDNA), $0.125 \mu \mathrm{l} 40 \mathrm{x}$ TaqMan assay and $2.5 \mu \mathrm{l}$ TaqMan genotyping master mix in a MicroAmp Optical 384 well reaction plate (Applied Biosystems) on a ViiA7 Real-Time PCR system (Applied Biosystems). Data were analysed by the sequence detection system (SDS) v. 2.3 (Applied Biosystems). Healthy control genotypes for the ASE analyses of ten of the samples were assessed previously [20], whereas the remaining four samples were genotyped as described above.

\section{Sensitivity assessment of allele-specific expression}

To assess the sensitivity of the allele-specific qPCR, gDNA homozygous for either allele of the corresponding SNPs were mixed in ratios of 4:1, 2:1, 1.5:1, 1.25:1, 1:1, $1: 1.25,1: 1.5,1: 2$ and 1:4. The ratios of allele-specific signals of these mixtures were quantified by real-time qPCR using TaqMan genotyping assays in TaqMan Universal Master Mix II, without UNG (Applied Biosystems), while including a heterozygous gDNA sample as a 1:1 ratio control of the alleles (Additional file 2: Figure S1). The samples were run in triplicate in a MicroAmp Optical 384 well reaction plate (Applied Biosystems) on ViiA7 Real-Time PCR system (Applied Biosystems), and data were analysed by SDS v. 2.3 (Applied Biosystems).

\section{Allele-specific expression}

For each cDNA sample from heterozygous donors, five replicate real-time qPCR measurements were performed using $5.66 \mathrm{ng}$ of cDNA in a $10 \mu \mathrm{l}$ reaction additionally containing $0.25 \mu \mathrm{l} 40 \mathrm{x}$ Primer Probe mix (Applied Biosystems), $5 \mu \mathrm{l}$ TaqMan Universal master mix II, without UNG (Applied Biosystems) and $4.15 \mu \mathrm{l}$ RNase-free water (Qiagen, Hilden, Germany). Genomic DNA (50 ng) from all heterozygous individuals was used to determine the technical difference in signals between alleles at an equal allele presence. The gDNA and cDNA samples were assayed using the same genotyping TaqMan primers and probes for the respective assays. All cDNA measurements were normalized against this technical difference (see data analyses and statistics below). Each plate included a negative control without cDNA and a no-RT control. The PCR reactions were performed in a MicroAmp optical 384 well reaction plate (Applied Biosystems) on the ViiA7 Real-Time PCR system (Applied
Biosystems), and data were analysed by SDS v. 2.3 software (Applied Biosystems).

\section{Quantitative real-time polymerase chain reaction of homozygous samples}

For each homozygous sample, cDNA generated from $6.52 \mathrm{ng}$ RNA was assayed in $10 \mu \mathrm{l}$ final volume containing $0.5 \mu \mathrm{l}$ of 20x Primer Probes (IKZF3: Hs00232635_m1 or IQGAP1: Hs00896595_m1, Applied Biosystems), 5 $\mu \mathrm{l} \mathrm{Taq-}$ Man Gene expression Master mix (Applied Biosystems) and 3.5 $\mu \mathrm{l}$ RNase-free water (Qiagen). A negative control without template and no-RT control were included in each plate. The reactions were performed in duplicates in a MicroAmp optical 384 well reaction plate (Applied Biosystems) on the ViiA7 Real-Time PCR system (Applied Biosystems), and data were analysed by SDS v. 2.3 software (Applied Biosystems). A standard curve was prepared from whole blood RNA from MS cases with a 1:2 fold dilution series (33.33-0.26 ng/ $\mu \mathrm{l})$. Gene expression was normalized relative to the housekeeping genes $18 \mathrm{~S}$ rRNA (4319413E), TBP (4326322E), and GAPDH (Hs03929097_g1). $18 S$ rRNA was selected as the preferential reference gene given its low variance in $\mathrm{Ct}$ between the different samples (data not shown).

\section{Peripheral blood mononuclear cells collection, cell lysis and Western blot analyses}

Whole blood samples from 32 healthy donors were collected among hospital employees and peripheral blood mononuclear cells were isolated by Lymphoprep (Axis Shield, Dundee, Scotland). Cells were resuspended in reducing SDS-loading buffer, sonicated and heated at $95{ }^{\circ} \mathrm{C}$ for $5 \mathrm{~min}$. Proteins from 250,000 cells were separated by SDS-polyacrylamide gel electrophoresis using pre-made Criterion gels (BioRad, Hercules, CA, USA) and transferred to polyvinylidene fluoride membrane (BioRad) using a Hoefer SemiPhor Semi-Dry transfer unit (Amersham Biosciences, Buckinghamshire, UK). The membrane was blocked in $3 \%$ skimmed milk in Tris-buffered saline (TBS, $\mathrm{pH}$ 7.4) containing $0.1 \%$ Tween-20 (Sigma Aldrich Corp., St Louis, MO, USA) (TBS-T) before incubation with antibodies, rabbit anti-IQGAP1 (ab133490, Abcam, Cambridge, UK) or mouse anti-GAPDH (6C5, sc-32233, Santa Cruz Biotechnology, Dallas, TX, USA). Bound antibodies were visualized by incubation with secondary horseradish peroxidase-conjugated goat anti-rabbit IgG or goat anti-mouse IgG (Jackson ImmunoResearch Laboratories Europe Ltd., Suffolk, UK) and ECL prime Western blotting detection reagent (GE Healthcare, Oslo, Norway ) and the ChemiDoc Touch Imaging System (BioRad). Densiometry of the Western blots was analysed by the ImageJ software [21]. 


\section{Data analyses and statistics}

Per donor in the ASE measurements, outlier values were excluded after inspection of box plots using SPSS (IBM SPSS Statistics v21.0, Chicago, IL, USA). Initially, measurements with extreme values were excluded, followed by generation of new box plots for the remaining data. Values marked as outlier values in the newly generated box plots were also excluded before analyses (for details on excluded measurements, see Additional file 3: Table S2). For each heterozygous donor, the relative allelic expression of the two alleles was expressed as delta cycle threshold $(\Delta \mathrm{Ct})=\mathrm{Ct}($ Allele2, FAM $)-C t$ (Allele1, VIC $)$. To account for technical differences between the used fluorophores, the $\Delta \mathrm{Ct}$ was normalized to the mean $\Delta \mathrm{Ct}$ of all gDNA (normalized $\Delta \mathrm{Ct}(\mathrm{n} \Delta \mathrm{Ct})=\Delta \mathrm{Ct}_{\mathrm{cDNA}}$ (per sample) $-\Delta \mathrm{Ct}_{\mathrm{gDNA}}$ (mean all samples). For each assessed SNP, a twotailed, unpaired Student's $t$-test was used to identify significant differences of the $\mathrm{n} \Delta \mathrm{Ct}$ per sample using the gDNA measurements grouped as the reference group. To test for differences on a global level, a two-tailed, unpaired Student's $t$-test was performed on all cDNA pooled against all gDNA pooled. Association between gene or protein expression and genotype was assessed by two-sided Mann-Whitney $U$-test using GraphPad Prism 6 (GraphPad Software, Inc., San Diego, CA, USA). $P$-values $<0.05$ were considered significant.

\section{Results}

\section{Patients and controls}

A total of 140 MS patients were available for the study, of whom 92 were heterozygous for at least one of the three SNPs studied; i.e. rs11052877 (CD69), rs907091 (IKZF3) and rs11609 (IQGAP1), and therefore included in the analyses. In Table 1 we summarize the characteristics of the patients and the healthy controls used in the AI measurements such as the male to female ratio, age at onset and fraction of oligoclonal band positive patients. The included patient group was representative of the Norwegian MS patient population [17]. Treatment data for the patients at the time of blood drawing was not available.
Selection of single nucleotide polymorphisms for allelespecific expression of multiple sclerosis candidate genes From the list of 110 non-HLA MS-associated SNPs [4, 5], we selected three SNPs for ASE analyses, based on the gene containing a transcribed SNP or suitable proxy SNP and the possible biological role of the gene in the immune system. The MS-associated SNP rs11052877 is located in a transcribed region of $C D 69$, whereas the remaining transcribed SNPs analysed; rs907091 in IKZF3 and rs11609 in IQGAP1, are in high LD with the MSassociated SNPs. The $r^{2}$ (D') of rs907091 to rs12946510 is $0.80(0.90)$, whereas the $r^{2}\left(D^{\prime}\right)$ of rs11609 to rs8042861 is $0.86(0.93)$.

\section{Consistent allelic imbalance is observed for rs907091 in IKZF3 and rs11609 in IQGAP1}

Among the 92 MS patients that were heterozygous for at least one of the three SNPs studied, 58 patients were heterozygous for rs11052877 (CD69), 30 patients were heterozygous for rs907091 (IKZF3) and 61 were heterozygous for rs11609 (IQGAP1). For each SNP, these samples were analysed for ASE by quantification of the alleles in cDNA generated from whole blood.

For the rs11052877 SNP in CD69, $52 \%$ of the samples carrying the MS risk allele displayed lower expression of the MS risk allele, whereas $26 \%$ of the samples displayed higher expression and the remaining $22 \%$ of the samples displayed no significant AI (Fig. 1a). This shows that there is no consistent cis-regulatory mechanism for CD69 associated with this SNP. Contrary to that, the transcript of the haplotype containing the MS risk allele at rs907091 in IKZF3, showed consistently higher expression level in all MS samples (Fig. 1b), whereas the transcript of the haplotype containing the MS risk allele at rs11609 in IQGAP1 was higher in the majority (90\%) of MS samples (Fig. 1c). For rs907091 in IKZF3, this difference corresponded to 1.17 in $\mathrm{n} \Delta \mathrm{Ct}$ value and to an expression ratio of the MS risk allele of 2.25:1. For rs11609 in IQGAP1, the $\mathrm{n} \triangle \mathrm{Ct}$ in the significant samples was 0.202 , which corresponds to an expression ratio of the risk allele versus non-risk allele of 1.15:1. When we compared all cDNA as a group against all gDNA as the

Table 1 Clinical and demographic information about the included MS patients and healthy controls

\begin{tabular}{|c|c|c|c|c|c|c|}
\hline \multirow[b]{3}{*}{ Variable } & & & \multicolumn{4}{|c|}{ Healthy controls } \\
\hline & \multicolumn{2}{|c|}{ MS patients } & \multicolumn{2}{|c|}{ ASE analyses } & \multicolumn{2}{|c|}{ Western blot analyses } \\
\hline & $n=$ & & $n=$ & & $n=$ & \\
\hline Male : Female ratio & 92 & $1: 3.4$ & 14 & $1: 4.7$ & 32 & $1: 1.5$ \\
\hline Age in years, mean (range) at sampling & 92 & $50(19-80)$ & 14 & $39(22-57)$ & 32 & $37(21-68)$ \\
\hline Age in years, mean (range) at disease onset & 89 & $35(14-60)$ & & & & \\
\hline Disease course at onset, fraction of relapsing-remitting MS & 86 & 0.87 & & & & \\
\hline Oligoclonal bands, positive fraction & 68 & 0.91 & & & & \\
\hline
\end{tabular}



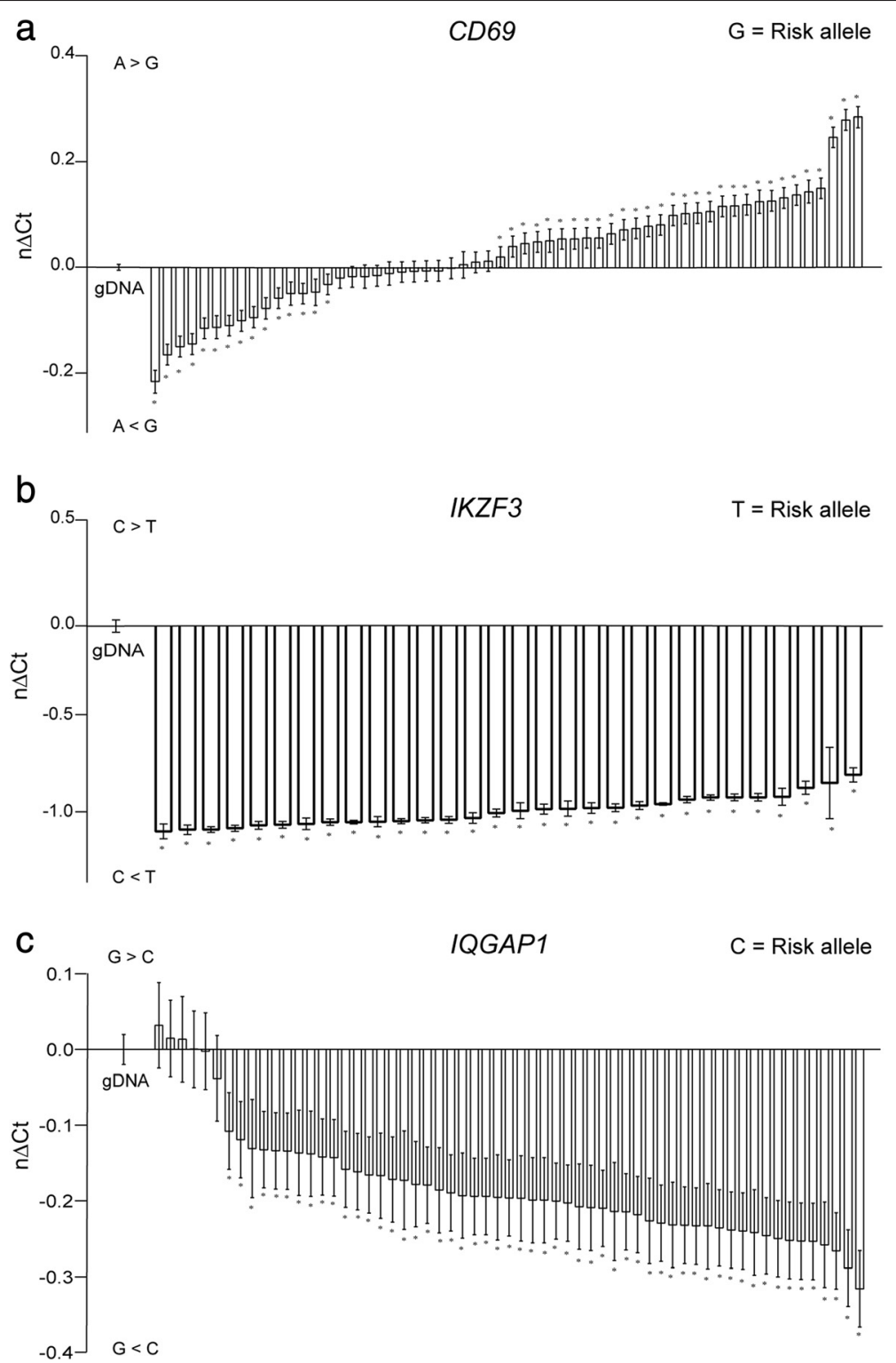

Fig. 1 Consistent allelic imbalance is observed for rs907091 in IKZF3 and rs11609 in IQGAP1. ASE analyses for (a) rs11052877 in CD69, (b) rs907091 in IKZF3, and (c) rs11609 in IQGAP1 were performed in samples from whole blood from MS patients heterozygous for the indicated SNPs. Each bar represents five replicate measurements. Data are presented as the normalized change in Ct between the two alleles (n$\Delta \mathrm{Ct})$. $\mathrm{n} \Delta \mathrm{Ct}$ values above zero represents lower expression of the MS risk allele, whereas $\mathrm{n} \Delta \mathrm{Ct}$ values below zero represent higher expression of the MS risk allele. Error bars represent the standard error of the mean. Unpaired Student's t-tests were used to compare each column with the gDNA measurement, $P$-values $<0.05$ were considered significant and are indicated with an asterisk. $A>B=$ allele $A$ expressed higher than $B, A<B=$ allele $A$ expressed lower than $B$

reference group, the significant differences in AI between these two groups persisted for $I K Z F 3$ as well as IQGAP1.

Since an incomplete LD $\left(\mathrm{r}^{2}=0.86, \mathrm{D}^{\prime}=0.93\right)$ exists between the studied SNP rs11609 and the reported MS associated SNP rs8042861 [5] in IQGAP1, we analysed whether the lack of AI for rs11609 in a small proportion of the samples could be due to absence of the MS-risk allele for these samples. However, all samples except one were also heterozygous for rs8042861 (data not shown), eliminating the possibility that absence of $\mathrm{AI}$ in the minority of the samples could be ascribed to a different genotype at the MS-risk SNP.

To unravel whether the consistent AI observed for IKZF3 could be attributed to the SNP most strongly associated to MS in this gene (rs12946510, which is in high, but incomplete LD with rs907091 $\left(\mathrm{r}^{2}=0.80\right.$, $\mathrm{D}^{\prime}=$ 0.90)), the samples were also analysed for double 
heterozygosity. We found that five of the samples were homozygous for the MS top hit rs12946510. This indicates that the observed $\mathrm{AI}$ is not driven by the genotype of the MS top hit rs12946510 in whole blood.

To exclude that the observed AI for rs907091 and rs11609 was limited to MS patients, we investigated ASE also in a smaller set of samples from healthy controls. We observed AI similar to the MS patients' samples indicating that the observed $\mathrm{AI}$ is independent of disease status (Additional file 4: Figure S2).

Altogether, these data indicate that the studied SNPs in IKZF3 and IQGAP1 are located in a cis-regulatory element, or that these SNPs mark a functional cis-regulatory element that impacts the per-allele transcript abundance in whole blood, independent of disease status.

\section{Samples from homozygous carriers of the risk allele at} rs11609 display higher IQGAP1 expression

To follow up the consistent AI of IKZF3 and IQGAPI observed in heterozygous individuals, we selected the samples that were homozygous for either allele of rs907091 (IKZF3) and rs11609 (IQGAP1) to determine whether the difference in allelic expression results in a difference in overall gene expression. We did not observe significant differences in IKZF3 gene expression between these two groups of homozygous for either the risk allele or protective allele (Fig. 2a). However, for $I Q G A P 1$, a significant genotype dependent expression was identified with higher expression in samples homozygous for the risk allele (Fig. 2b), in line with our ASE analyses described above. To further investigate whether higher IQGAP1 for the MS risk allele results in a higher IQGAP1 protein level, we measured this expression by
Western blotting in lysates from peripheral blood mononuclear cells from healthy individuals homozygous for either the risk or protective rs11609 allele at IQGAP1. Although we observe a slightly higher IQGAP1 expression in samples homozygous for the risk allele, this did not reach significance (Additional file 5: Figure S3).

\section{Discussion}

Recent large-scale genotyping studies in MS have revealed more than 110 genetic variants associated with MS $[4,5]$, however, the functional impact of the majority of these SNPs remains unknown and needs to be determined experimentally. To this end, we investigated the ASE of three transcribed SNPs on MS susceptibility haplotypes in whole blood of MS patients and show AI for the SNPs rs907091 and rs11609, which are transcribed in IKZF3 and IQGAP1, respectively. Furthermore, we observed a significantly higher level of IQGAP1 gene expression for samples homozygous for the allele with a relatively higher expression in the ASE measurements, possibly indicating that the allelic imbalance persists beyond the relative expression of the alleles. Given this observation, a relative higher expression of IQGAP1 for carriers of the minor allele might contribute to an increase in MS susceptibility.

Allele-specific qPCR is a sensitive tool for evaluation of cis-acting regulatory polymorphisms and can directly detect differences in allelic output when assessed in heterozygous carriers. By examining the relative expression levels of two alleles within the same biological sample, variations introduced by the environment or differences in physiological background of the individuals enrolled in the study were minimized. AI measurements are relatively robust for detecting and quantifying variations in
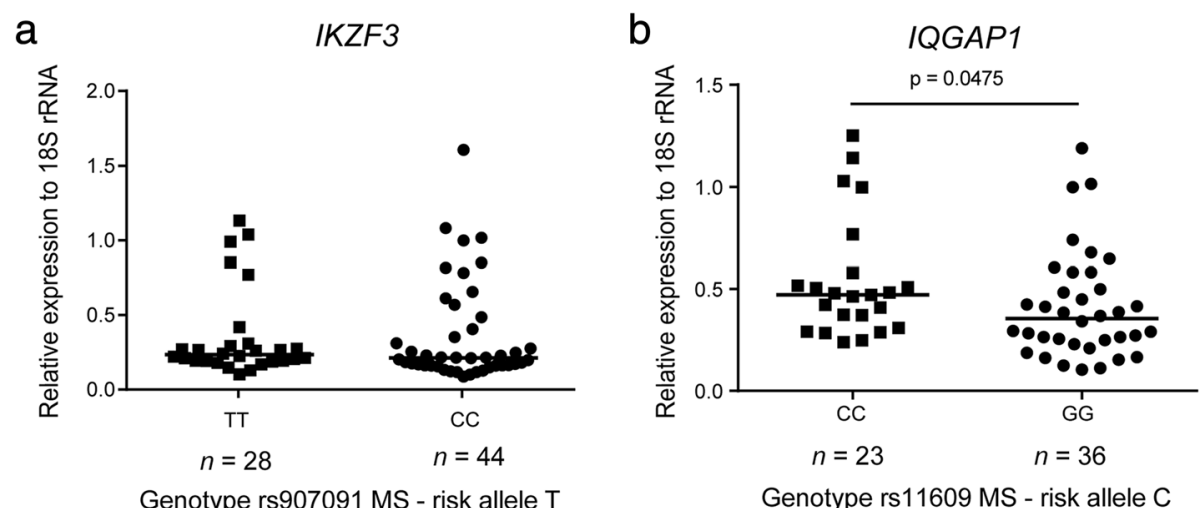

Fig. 2 Samples from homozygous carriers of the risk allele at rs11609 display higher IQGAP1 expression. Expression of IKZF3 and IQGAP1 relative to 18 S rRNA in samples from MS patients genotyped for (a) rs907091 in IKZF3 (TT (risk): $n=28$; CC (protective): $n=44$ ) and (b) rS1 1609 IQGAP1 (CC (risk): $n=23$; GG (protective): $n=36$ ). Samples from individuals homozygous for the risk allele were compared with samples from individuals homozygous for the protective allele with two-tailed Mann-Whitney U-test. P-values $<0.05$ were considered significant, only the significant $p$-value is provided in the graph 
allelic expression. However, the AI observed in the MS cases for IKZF3 and IQGAP1 was also observed in healthy controls, indicating that MS itself does not impact the AI of these genes.

It is important to note that the overall expression of a gene can be influenced by multiple regulatory mechanisms. Here we have a specific focus on those genetic loci that were shown to have an association to MS. The rs11609 SNP in IQGAP1 has been identified as an eQTL for IQGAP1 in many tissues (for instance mammary, adipose, thyroid, stomach and skin tissue), however not in whole blood. In our study, we observe relatively higher allelic expression of the MS-risk allele at rs11609, which is consistent with higher overall gene expression of IQGAP1 in samples homozygous for the risk allele. This is similar to data from other tissues in the GTEx data where rs11609 is reported as an eQTL with higher expression for carriers of the minor allele. Our data indicate that also in whole blood, the haplotype on which rs11609 resides has cis-regulatory properties affected by genetic variation. We found double heterozygosity for all samples except one for rs8042861 and rs11609 in IQGAP1. The observed AI for rs11609 may originate from different mechanisms of IQGAP1 gene regulation. Interestingly, rs11609 is situated in an open chromatin region (genome.ucsc.edu), making it a plausible candidate for influencing gene expression. However, there are several SNPs in high LD with the MS associated rs8042861 SNP that are within transcription factor binding sites (genome.ucsc.edu) and thereby might be the causal SNP mediating the observed effect. Indeed, it has been suggested that for most of the complex diseaseassociated SNPs, the underlying SNP is predicted to be located within the LD block of the associated SNP [14]. Further studies of genetic variation present in this region of interest for functional properties are necessary to better discern among these possibilities. The exact molecular mechanism of how higher IQGAP1 expression may contribute to increased MS risk is unclear and warrants more investigation. IQ Motif Containing GTPase Activating Protein 1 (IQGAP1) is a ubiquitously expressed protein, belonging to the scaffolin-family, and has emerged as a critical regulator of several signalling pathways in a variety of cell types, including immune cells, where it plays important roles for cytoskeletonmediated processes [22]. IQGAP1 has shown to be instrumental for leukocyte chemotaxis and natural killer (NK)-cell cytotoxicity [23, 24]. Higher IQGAP1 expression, as observed from MS risk loci, would lead to aberrant leukocyte cell migration and NK cell activity, and could thereby contribute to MS disease. Furthermore, defects of the IQGAP1-interacting protein, Rap1b, result in multiple NK and B-cell dysfunctions $[25,26]$, indicating that a change in IQGAP1 expression in those immune cells could also have an impact for MS development. Although we observed higher IQGAP1 protein expression in samples homozygous for the risk allele, this did not reach statistical significance. To reach $80 \%$ power to detect a difference of this magnitude with this standard deviation, a sample size of at least 43 in each group would be required (Additional file 6).

In agreement with the GTEx database (accessed March 2016), we did not observe consistent allelic imbalance for the CD69 gene, indicating that the SNP association to MS may not be attributable to cis-regulatory properties in whole blood. Whether there are such cisregulatory properties for this SNP in more specialized cell sub-types within the whole blood or other cells relevant to MS remains to be investigated. However, in line with our results, recent bioinformatic analyses indicated that the genotype of the MS associated rs11052877 SNP does not affect any transcription factor DNA binding motifs [27].

The rs907091 SNP in IKZF3 has been shown to be an eQTL influencing the expression or stability of the gene transcript in lymphoblastoid cell lines [14, 15], but not in whole blood (GTEx, accessed March 2016). The consistent AI observed for rs907091 indicates that this SNP potentially has eQTL properties in whole blood. We did not observe differences of overall IKZF3 gene expression between the homozygous carriers of the minor and major allele, indicating that the eQTL properties may be most pronounced in homogeneous samples. Alternatively, our study may have a limited sample size and therefore lack the power to detect such differences in whole blood. Furthermore, the blood samples used in this study are from an MS patient population at different disease stages and using different treatments. This heterogeneity within the sampled patients could contribute to the lack of differential gene expression in samples sorted on different genotypes.

It has previously been suggested that most disease variants exert subtle and highly context-dependent effects on gene regulation [14], therefore we cannot exclude that using a comparable number of samples from specific immune cell subsets could detect IKZF3 expression differences depending on the SNP genotype. The SNP rs907091 is located in the 3' untranslated region of the IKZF3 gene encoding AIOLOS, an Ikaros transcription factor involved in regulation of lymphocyte development [28]. IKZF3 deficiency in mice mainly affects B cell function [29], giving rise to hyperactive immature B cell precursors [30,31], and decreased peritoneal, marginal and recirculating B cells $[30,32,33]$. An increase in IKZF3 expression in B cells might lead to aberrant B-cell responses that could play a role for MS development. 
Since there is a lack of complete double heterozygosity between the AI SNP and the MS top-hit SNP rs12946510 in IKZF3, the consistent AI observed for rs907091 cannot be solely attributed to the genotype of the MS-associated SNP. The mechanisms through which the rs12946510 might have functional consequences for MS development need further investigations. Moreover, the probabilistic identification of causal SNPs fine mapping algorithm from the Broad Institute [14] indicates that a large array of SNPs may be underlying the identified MS association signal, including rs907091, without a clear indication for the actual functional SNP at this locus. Therefore, the IKZF3 AI can be driven by multiple SNPs without any of these having a probability above $20 \%$ of being the true functional SNP. Previous experimental studies have indicated that polymorphisms in IKZF3 influence susceptibility for development of several autoimmune disorders in addition to MS [14, 34-38]. Further investigations into how perturbation of the investigated genes in whole blood or in more specific cell subtypes are needed to further elucidate how the SNPs cis-regulation may contribute to MS risk.

\section{Conclusions}

This study showed a molecular mechanism, which might contribute to the MS-association of two SNPs. We identified differences in allelic output and/or overall gene expression for rs907091 in IKZF3 and rs11609 in IQGAP1, which are both in high LD with MS-associated SNPs. Additional studies into the functional properties of these SNPs and the impact on the protein expression in specific cell types that are present in whole blood may identify which cells drive the association of these SNPs to MS development. Furthermore, since these SNPs reside in large LD blocks that could harbour one or more true functional variants, additional investigations to identify the true functional variations are warranted.

\section{Ethics (and consent to participate)}

The Regional Committee for Medical and Health Research Ethics South East, Norway, approved this study. Signed informed consent was obtained from all study participants.

\section{Consent to publish \\ Not applicable.}

\section{Availability of data and materials}

The datasets supporting the conclusions of this article are included in Additional file 7.

\section{Additional files}

Additional file 1: Table S1. PCR primers used to verify gene expression of SNP-containing regions of the indicated genes in whole blood. (PDF $133 \mathrm{~kb}$ )

Additional file 2: Figure S1. Assay sensitivity testing for allelespecific expression with known ratios of alleles. Allele-specific qPCR was performed on mixtures of known ratios of gDNA obtained from samples homozygous for either allele of rs11052877, rs907091 or rs11609, respectively. Mixtures were prepared with allele ratios of $4: 1$, $2: 1,1.5: 1,1.25: 1,1: 1,1: 1.25,1: 1.5,1: 2$ and 1:4. As control for a 1:1 allele ratio, a heterozygous sample $(H)$ was included for each assay. Data is expressed as the mean of three measurements and error bars represent the standard error of the mean. (TIF $1553 \mathrm{~kb}$ )

Additional file 3: Table S2. Details of excluded measurements per investigated gene. (PDF $82 \mathrm{~kb}$ )

Additional file 4: Figure S2. Allele-specific expression analyses of IKZF3 and IQGAP1 in samples from healthy controls. Allele-specific expression of the genes was normalised to the mean of all genomic DNA for (a) rs907091 in IKZF3 and (b) rs11609 in IQGAP1. Each bar represents five replicate measurements. Data are presented as the normalized change in $\mathrm{Ct}$ between the two alleles ( $\mathrm{n} \Delta \mathrm{Ct}$ ). $\mathrm{n} \Delta \mathrm{Ct}$ values above zero represent lower expression of the MS risk allele, whereas $n \Delta C t$ values below zero represents higher expression of the MS risk allele. Error bars represent the standard error of the mean. A two-tailed unpaired Student's t-test was used to compare each column with the gDNA measurement,

$P$-values $<0.05$ are indicated with ${ }^{*}$. $A>B=$ allele $A$ expressed higher than $B, A<B=$ allele $A$ expressed lower than $B$. (TIF 84 kb)

Additional file 5: Figure S3. IQGAP1 protein expression in peripheral blood mononuclear cells from healthy controls. (a) Whole-cell lysates from peripheral blood mononuclear cells from healthy controls genotyped for rs11609 in IQGAP1 (CC (risk): $n=6$; GG (protective): $n=9$ ) were immunoblotted with indicated antibodies. (b) Bands were quantified and normalized with GAPDH as described in Materials and Methods. The graph shows the mean with standard error of the mean. A two- sided Mann-Whitney U-test was performed to compare the groups. (TIF $1357 \mathrm{~kb}$ )

Additional file 6: Power analyses of IQGAP1 protein expression. (PDF $141 \mathrm{~kb}$ )

Additional file 7: Raw dataset supporting the conclusions of this article. (XLSX $31 \mathrm{~kb})$

\section{Abbreviations}

ASE: allele-specific expression; CD69: cluster of differentiation 69; CDNA: complementary DNA; CNS: central nervous system; Ct: cycle threshold; eQTL: expression quantitative trait loci; gDNA: genomic DNA; GWAS: genome - wide association study; HLA: human leukocyte antigen; IKZF3: IKAROS family zinc finger 3; IQGAP1: IQ motif containing GTPase activating protein 1; LD: linkage disequilibrium; MAF: minor allele frequency; MS: multiple sclerosis; NK: natural killer; qPCR: quantitative polymerase chain reaction; RT: reverse transcription; SNPs: single nucleotides polymorphisms.

\section{Competing interests}

The authors declare that they have no competing interests.

\section{Authors' contributions}

Conceived and designed the experiments: PKK, HFH, SDB and TB. Performed experiments: PKK and TB. Collection of samples: PKK, HFH, TB, SDB, KMM and JHA. Data analysis, results interpretation and discussion: PKK, HFH, SDB and TB. Figure compositions: PKK, SDB and TB. Manuscript writing and revision: PKK, HFH, $\mathrm{SDB}, \mathrm{TB}, \mathrm{KMM}$ and $\mathrm{JHA}$. All authors read and approved the final manuscript.

\section{Acknowledgements}

We thank all patients and controls, research nurses and doctors involved in the collection of samples included in this study. We acknowledge engineers at the Institute of Public Health and Anja Bjølgerud and Gun Berge for handling of samples and the Norwegian MS Registry and Biobank for collaboration. 


\section{Funding}

This study was funded by The South-Eastern Norway Regional Health Authority, the Norwegian Research Council and the Odd Fellow Society. The funders had no role in the design of the study and collection, analysis, decision to publish, interpretation of data or preparation of the manuscript.

\section{Author details}

${ }^{1}$ Department of Neurology, Oslo University Hospital, Oslo, Norway. ${ }^{2}$ Institute of Clinical Medicine, University of Oslo, Oslo, Norway. ${ }^{3}$ Norwegian Multiple Sclerosis Registry and Biobank, Department of Neurology, Haukeland University Hospital, Bergen, Norway. ${ }^{4} \mathrm{KG}$ Jebsen Centre for MS-research, Department of Clinical Medicine, University of Bergen, Bergen, Norway.

\section{Received: 2 October 2015 Accepted: 7 April 2016}

\section{Published online: 14 April 2016}

\section{References}

1. Compston A, Coles A. Multiple sclerosis. Lancet. 2008;372(9648):1502-17.

2. Gourraud PA, Harbo HF, Hauser SL, Baranzini SE. The genetics of multiple sclerosis: an up-to-date review. Immunol Rev. 2012;248(1):87-103.

3. Jersild C, Svejgaard A, Fog T. HL-A antigens and multiple sclerosis. Lancet. 1972;1(7762):1240-1.

4. International Multiple Sclerosis Genetics Consortium, Wellcome Trust Case Control Consortium 2, Sawcer S, Hellenthal G, Pirinen M, Spencer CC, Patsopoulos NA, Moutsianas L, Dilthey A, Su Z, et al. Genetic risk and a primary role for cell-mediated immune mechanisms in multiple sclerosis. Nature. 2011:476(7359):214-9.

5. International Multiple Sclerosis Genetics Consortium, Beecham AH, Patsopoulos NA, Xifara DK, Davis MF, Kemppinen A, Cotsapas C, Shah TS, Spencer C, Booth D, et al. Analysis of immune-related loci identifies 48 new susceptibility variants for multiple sclerosis. Nat Genet. 2013;45(11):1353-60.

6. International Multiple Sclerosis Genetics Consortium. Network-based multiple sclerosis pathway analysis with GWAS data from 15,000 cases and 30,000 controls. Am J Hum Genet. 2013;92(6):854-65.

7. Cookson W, Liang L, Abecasis G, Moffatt M, Lathrop M. Mapping complex disease traits with global gene expression. Nat Rev Genet. 2009:10(3): 184-94.

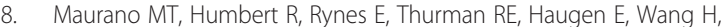
Reynolds AP, Sandstrom R, Qu H, Brody J, et al. Systematic localization of common disease-associated variation in regulatory DNA. Science. 2012; 337(6099):1190-5.

9. Disanto G, Kjetil Sandve G, Ricigliano VA, Pakpoor J, Berlanga-Taylor AJ, Handel AE, Kuhle J, Holden L, Watson CT, Giovannoni G, et al. DNase hypersensitive sites and association with multiple sclerosis. Hum Mol Genet. 2014:23(4):942-8.

10. GTEx Consortium. Human genomics. The Genotype-Tissue Expression (GTEx) pilot analysis: multitissue gene regulation in humans. Science. 2015; 348(6235):648-60.

11. Cao Y, Goods BA, Raddassi K, Nepom GT, Kwok WW, Love JC, Hafler DA. Functional inflammatory profiles distinguish myelin-reactive $T$ cells from patients with multiple sclerosis. Sci Transl Med. 2015;7(287):287ra274.

12. Ratzer R, Sondergaard HB, Christensen JR, Bornsen L, Borup R, Sorensen PS, Sellebjerg F. Gene expression analysis of relapsing-remitting, primary progressive and secondary progressive multiple sclerosis. Mult Scler. 2013; 19(14):1841-8.

13. Lo HS, Wang Z, Hu Y, Yang HH, Gere S, Buetow KH, Lee MP. Allelic variation in gene expression is common in the human genome. Genome Res. 2003; 13(8):1855-62

14. Farh KK, Marson A, Zhu J, Kleinewietfeld M, Housley WJ, Beik S, Shoresh N, Whitton H, Ryan RJ, Shishkin AA et al. Genetic and epigenetic fine mapping of causal autoimmune disease variants. Nature. 2015;518(7539):337-43.

15. Lappalainen T, Sammeth M, Friedlander MR, t Hoen PA, Monlong J, Rivas MA, Gonzalez-Porta M, Kurbatova N, Griebel T, Ferreira PG, et al. Transcriptome and genome sequencing uncovers functional variation in humans. Nature. 2013;501(7468):506-11.

16. Myhr KM, Grytten N, Aarseth J. The Norwegian multiple sclerosis registry and biobank. Acta Neurol Scand. 2012;126(s195):20-3.

17. Berg-Hansen $P$, Moen SM, Harbo HF, Celius EG. High prevalence and no latitude gradient of multiple sclerosis in Norway. Mult Scler. 2014;20(13):1780-2.
18. Johnson AD, Handsaker RE, Pulit SL, Nizzari MM, O'Donnell CJ, de Bakker PI. SNAP: a web-based tool for identification and annotation of proxy SNPS using HapMap. Bioinformatics. 2008;24(24):2938-9.

19. Riva A, Kohane IS. SNPper: retrieval and analysis of human SNPS. Bioinformatics. 2002;18(12):1681-5.

20. Bos SD, Page CM, Andreassen BK, Elboudwarej E, Gustavsen MW, Briggs F, Quach H, Leikfoss IS, Bjolgerud A, Berge T, et al. Genome-wide DNA methylation profiles indicate CD8+ T cell hypermethylation in multiple sclerosis. PLoS One. 2015;10(3):e0117403.

21. Schneider CA, Rasband WS, Eliceiri KW. NIH Image to ImageJ: 25 years of image analysis. Nat Methods. 2012;9(7):671-5.

22. Abel AM, Schuldt KM, Rajasekaran K, Hwang D, Riese MJ, Rao S, Thakar MS, Malarkannan S. IQGAP1: Insights into the function of a molecular puppeteer. Mol Immunol. 2015;65(2):336-49.

23. Neel NF, Sai J, Ham AJ, Sobolik-Delmaire T, Mernaugh RL, Richmond A. IQGAP1 is a novel CXCR2-interacting protein and essential component of the "chemosynapse". PLoS One. 2011;6(8):e23813.

24. Bamidele AO, Kremer KN, Hirsova P, Clift IC, Gores GJ, Billadeau DD, Hedin KE. IQGAP1 promotes CXCR4 chemokine receptor function and trafficking via EEA-1+ endosomes. J Cell Biol. 2015:210(2):257-72.

25. Chu H, Awasthi A, White 2nd GC, Chrzanowska-Wodnicka M, Malarkannan S. Rap $1 \mathrm{~b}$ regulates $\mathrm{B}$ cell development, homing, and T cell-dependent humoral immunity. J Immunol. 2008;181(5):3373-83.

26. Awasthi A, Samarakoon A, Chu H, Kamalakannan R, Quilliam LA Chrzanowska-Wodnicka M, White GC, 2nd, Malarkannan S. Rap1b facilitates NK cell functions via IQGAP1-mediated signalosomes. J Exp Med. 2010; 207(9):1923-38

27. Briggs FB, Leung $\sqcup$, Barcellos LF. Annotation of functional variation within non-MHC MS susceptibility loci through bioinformatics analysis. Genes Immun. 2014;15(7):466-76.

28. Cortes M, Wong E, Koipally J, Georgopoulos K. Control of lymphocyte development by the Ikaros gene family. Curr Opin Immunol. 1999;11(2):167-71.

29. John $L B$, Ward $A C$. The Ikaros gene family: transcriptional regulators of hematopoiesis and immunity. Mol Immunol. 2011;48(9-10):1272-8.

30. Wang JH, Avitahl N, Cariappa A, Friedrich C, Ikeda T, Renold A, Andrikopoulos K, Liang L, Pillai S, Morgan BA, et al. Aiolos regulates B cell activation and maturation to effector state. Immunity. 1998:9(4):543-53.

31. Sun J, Matthias G, Mihatsch MJ, Georgopoulos K, Matthias P. Lack of the transcriptional coactivator OBF-1 prevents the development of systemic lupus erythematosus-like phenotypes in Aiolos mutant mice. J Immunol. 2003;170(4):1699-706.

32. Cariappa A, Tang M, Parng C, Nebelitskiy E, Carroll M, Georgopoulos K, Pillai $\mathrm{S}$. The follicular versus marginal zone B lymphocyte cell fate decision is regulated by Aiolos, Btk, and CD21. Immunity. 2001;14(5):603-15.

33. Narvi E, Nera KP, Terho P, Mustonen L, Granberg J, Lassila O. Aiolos controls gene conversion and cell death in DT40 B cells. Scand J Immunol. 2007; 65(6):503-13.

34. Kurreeman FA, Stahl EA, Okada Y, Liao K, Diogo D, Raychaudhuri S, Freudenberg J, Kochi Y, Patsopoulos NA, Gupta N, et al. Use of a multiethnic approach to identify rheumatoid- arthritis-susceptibility loci, 1 p36 and 17q12. Am J Hum Genet. 2012;90(3):524-32.

35. Cai X, Qiao Y, Diao C, Xu X, Chen Y, Du S, Liu X, Liu N, Yu S, Chen D, et al. Association between polymorphisms of the IKZF3 gene and systemic lupus erythematosus in a Chinese Han population. PLoS One. 2014;9(10):e108661.

36. Marinho S, Custovic A, Marsden P, Smith JA, Simpson A. 17q12-21 variants are associated with asthma and interact with active smoking in an adult population from the United Kingdom. Ann Allergy Asthma Immunol. 2012; 108(6):402-411.e409.

37. Lessard CJ, Adrianto I, Ice JA, Wiley GB, Kelly JA, Glenn SB, Adler AJ, Li H, Rasmussen A, Williams AH, et al. Identification of IRF8, TMEM39A, and IKZF3ZPBP2 as susceptibility loci for systemic lupus erythematosus in a largescale multiracial replication study. Am J Hum Genet. 2012;90(4):648-60.

38. Qiu R, Zhang H, Zhao H, Li J, Guo C, Gong Y, Liu Q. Genetic variants on $17 q 21$ are associated with ankylosing spondylitis susceptibility and severity in a Chinese Han population. Scand J Rheumatol. 2013:42(6):469-72. 